\title{
Efecto cinemático durante la marcha de la modificación medial sobre ortesis plantares de la técnica de adaptación en directo en el pie plano flexible infantil: a propósito de un caso clínico
}

Kinematic effect during walking of medial modification on plantar orthoses of direct adaptation technique on a child whit flexible flat foot: purpose of a clinical case

Moisés Pardos Barradoํy Daniel García García²

${ }^{1}$ Consulta privada. Zaragoza, España. ${ }^{2}$ Consulta privada. Huesca, España

Palabras clave:

Técnica de adaptación en directo (TAD), técnica Medial Heel Skive (TMHS), pie plano flexible infantil, análisis cinemático de la marcha, técnica Lateral Heel Skive (TLHS).

\section{Resumen}

Se presenta una modificación de la Técnica de Adaptación en Directo (TAD), en el tratamiento conservador ortésico en un caso clínico de pie plano flexible infantil. En este caso clínico se comparó la eficacia de la ortesis de resina realizada con la TAD tradicional con una ortesis realizada mediante la modificación MEDIAL TAD. Esta modificación consiste en la creación de una cuña supinadora intrínseca en la ortesis de resina, y se presentan dos opciones para ello. Para la cuantificación del efecto se utilizó un sistema de captura del movimiento de tipo óptico, constatándose en ambos pies un mayor control de pronación con la plantilla obtenida a partir de la TAD modificada. Los beneficios de esta modificación podrían abarcar desde un pie pronado con un MEDIAL TAD hasta un pie supinado con un LATERAL TAD.
Keywords:

Direct Adaptation Technique (TDA), Medial Heel Skive Technique (TMHS), flexible flatfoot in children, kinematic gait analysis, Lateral Heel Skive Technique (TLHS)

\section{Abstract}

A modification of the Direct Adaptation Technique (TAD) is presented, for the conservative orthotic treatment in a clinical case of pediatric flexible flatfoot. In this clinical case, the efficacy of the resin orthosis made with the traditional TAD was compared with an orthosis made using the MEDIAL TAD modification. This modification consists of the creation of an intrinsic supination wedge in the resin orthosis, and two options are presented for this modification. To quantify the effect, an optical type motion capture system was used to assess its effect with a greater pronation control in both feet with the template obtained from the modified TAD. The benefits of this modification could range from a pronated foot with a MEDIAL TAD to a supinated foot with a LATERAL TAD. 


\section{INTRODUCCIÓN}

La técnica de adaptación en directo, descrita en el año 1994 por Tomás Céspedes, Adelina Dorca y cols., permitía obtener la ortesis plantar a través de la aplicación del material directamente sobre el pie del paciente, reduciendo y simplificando el proceso de confección del tratamiento ortopodológico'.

Pardos, en el año 2018², realizó un estudio comparativo del efecto reductor de la pronación de la articulación subta$\operatorname{lar}($ AST) y mediotarsiana (AMT), utilizando el sistema Natural Point OptiTrack de análisis cinemático de tipo óptico, considerado de alta precisión para Tewlis y cols. comparado con el sistema Vicon ${ }^{3}$, gold standard de los análisis cinemáticos según

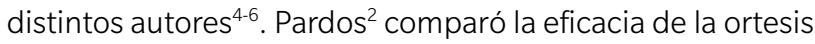
de resina mediante TAD con una ortesis invertida de Blake (OIB) de polipropileno y una ortesis por elementos de Lelièvre. En aquella investigación ${ }^{2}$ se concluyó que las ortesis por elementos (OE) y polipropileno (OP) corrigieron significativamente más el rango eversor de retropié que la ortesis de resina (OR). Asimismo, el valor de máxima eversión se redujo significativamente al introducir la ortesis en el calzado, siendo esta reducción significativamente mayor con las ortesis por elementos y de polipropileno respecto de la de resina. Las tres ortesis redujeron entre $3^{\circ}$ y $4^{\circ}$ el valor de máxima eversión de acuerdo con los resultados obtenidos por McCulloch y cols. ${ }^{7}$ y superiores a los $2.3^{\circ}$ reportados por Mündermann y cols. ${ }^{8}$, $2.2^{\circ}$ de Genova y Gross ${ }^{9}, 1-3^{\circ}$ de Eng y Pierrynowsky ${ }^{10}, 1.59^{\circ}$ de Williams y cols. ${ }^{11}$ o los $1.5^{\circ}$ de Nigg y cols. ${ }^{12}$. Respecto de la AMT, el calzado redujo significativamente el rango eversor de AMT respecto de la forma de caminar descalzo. Aunque la ortesis de resina registró la mayor reducción del rango eversor, no se detectaron diferencias entre las tres ortesis comparadas y la situación de caminar calzado sin ortesis. El calzado respecto del modo descalzo y las ortesis respecto del calzado sin ortesis redujeron significativamente el valor de máxima eversión de la AMT en plano frontal. Estos resultados serían coherentes por los reportados por Moraleda y Mubarak ${ }^{13}$, Sinha y cols. ${ }^{14}$ o Boks y cols. ${ }^{15}$. Aunque no hubo diferencias entre la OE y OR, ambas redujeron la eversión significativamente más que la OP.

A mediados de los años 80, el Dr. Richard Blake ${ }^{16}$, insatisfecho con los resultados que obtenía para el control de la pronación en algunos pies pronados con las ortesis funcionales tipo Root ${ }^{17}$, comenzó a idear un nuevo sistema de realización de ortesis caracterizado por una mayor inversión de los tratamientos más orientados al enfoque antiguo de Whitman ${ }^{18}$ y Roberts ${ }^{19}$. Blake desarrolló lo que hoy se conoce como la ortesis invertida de Blake (OIB), utilizada y aceptada internacionalmente durante los últimos 30 años $^{20}$ y que básicamente consiste en la obtención de un molde positivo de la planta del pie, como hacía Root ${ }^{17}$, pero realizando un giro en inversión del molde $\left(15^{\circ}, 20^{\circ}\right.$ o incluso más) para adaptar en esa posición la ortesis. Kirby y Green ${ }^{21}$ afirmaron en 1992 que la razón por la que la OIB era más eficiente que la clásica ortesis funcional para el control excesivo de la pronación era que la forma invertida de la cazoleta de talón redirigía las fuerzas de reacción del suelo hacia los aspectos mediales generando mayores momentos supinadores sobre la AST. Afirmación corroborada por investigaciones posteriores como la de Williams y cols. en el año 2003, en la que se constató una reducción significativa del momento eversor del retropié al comparar la situación de correr con OIB respecto correr con ortesis funcional, y sin ortesis funcional ${ }^{11}$.

Kirby observó la eficacia de la OIB en adultos, pero no obtenía los mismos resultados con los niños con pie plano flexible. Kirby señaló que el perfil redondeado de la cara plantar del talón de los niños podría ser la razón por la que la OIB a menudo no funciona tan bien en los niños como en los adultos, con un perfil de la cara plantar de talón aplanado, probablemente fruto del moldeado de la grasa plantar por efecto del envejecimiento y las fuerzas de compresión ${ }^{6}$. Esta observación le llevó a plantear una modificación del molde positivo, describiendo en 1992 la técnica Medial Heel Skive (TMHS) ${ }^{22}$.

El presente artículo presenta una modificación de la TAD, consistente en la introducción de la TMHS dentro de la plantilla realizada con la TAD, aplicada a un niño de 11 años, diagnosticado de pie plano flexible que durante dos años había sido tratado con una ortesis confeccionada con la TAD tradicional.

\section{CASO CLÍNICO}

Niño de 11 años de edad que acude a consulta para revisión de pie plano flexible. Se presenta asintomático, sin antecedentes personales referidos por la madre. Presenta genu valgo sin monitorizar. No se observan dismetrías aparentes. El ángulo pierna pie en flexión dorsal de tobillo y rodilla extendida es de $90^{\circ}$ y desciende a $80^{\circ}$ con la flexión de rodiIla, de forma bilateral. La resistencia a la dorsiflexión de primer metatarsiano está disminuida. Aunque se observó un subjetivo aumento de la laxitud, la escala de Beigthon fue inferior a 4.

Acude por primera vez a la consulta con 9 años derivado del pediatra para valorar la instauración de tratamiento ortésico, con un IMC de 18.29 p80 [146 cm/39 kg]. Durante dos años utilizó ortesis plantar de resina confeccionada con la TAD tradicional ${ }^{1}$, pasando su IMC a 25.4 p99 [155 cm/61 kg]. Los test funcionales, Single Heel Rise Test (SHRT), Double Heel Rise Test(DHRT) y Test de Jack, continuaron siendo negativos, descartando aparentemente el carácter rígido de la disfunción. Se ha observado una mejoría subjetiva en el alineamiento de retropié de forma bilateral con la posición relajada de calcáneo en apoyo PRCA derecho de 9 a $7^{\circ}$ y PRCA izquierdo de 10 a $8^{\circ}$. También ha mejorado su índice de postura del pie (IPP), pasando en pie derecho de $+10 \mathrm{a}+9$, y de $+11 \mathrm{a}+10$ en izquierdo.

Al tratarse de un menor, los padres del niño que participó consintieron de manera expresa la realización de la prueba y el uso de los resultados en investigación clínica. 

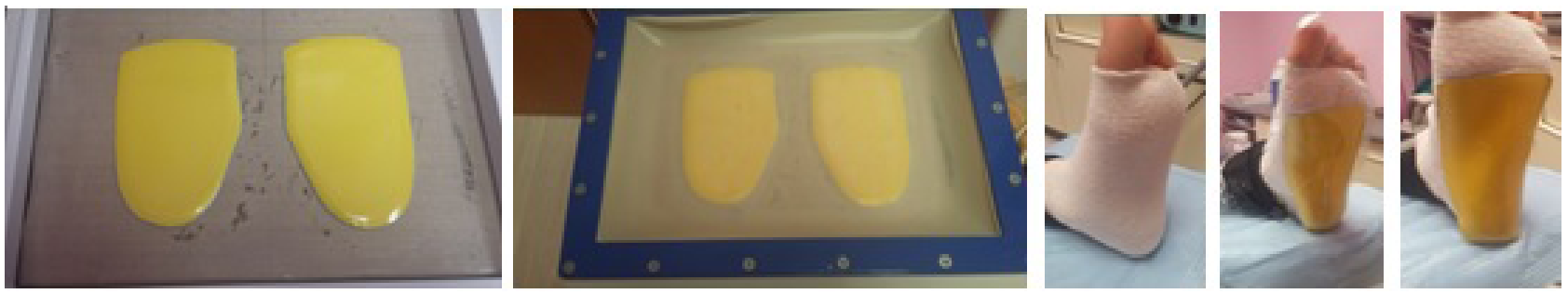

Figura 1. De izquierda a derecha, técnica TAD en diferentes fases de la adaptación.

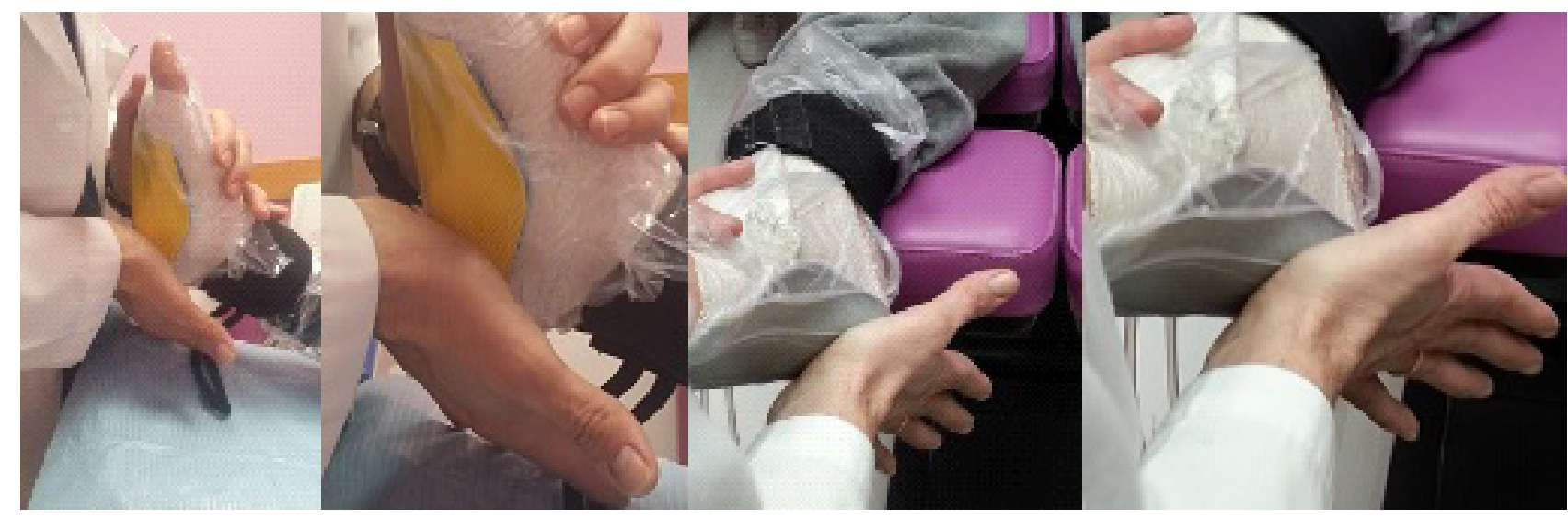

Figura 2. Maniobra de adaptación del medial TAD. Modificación manual.
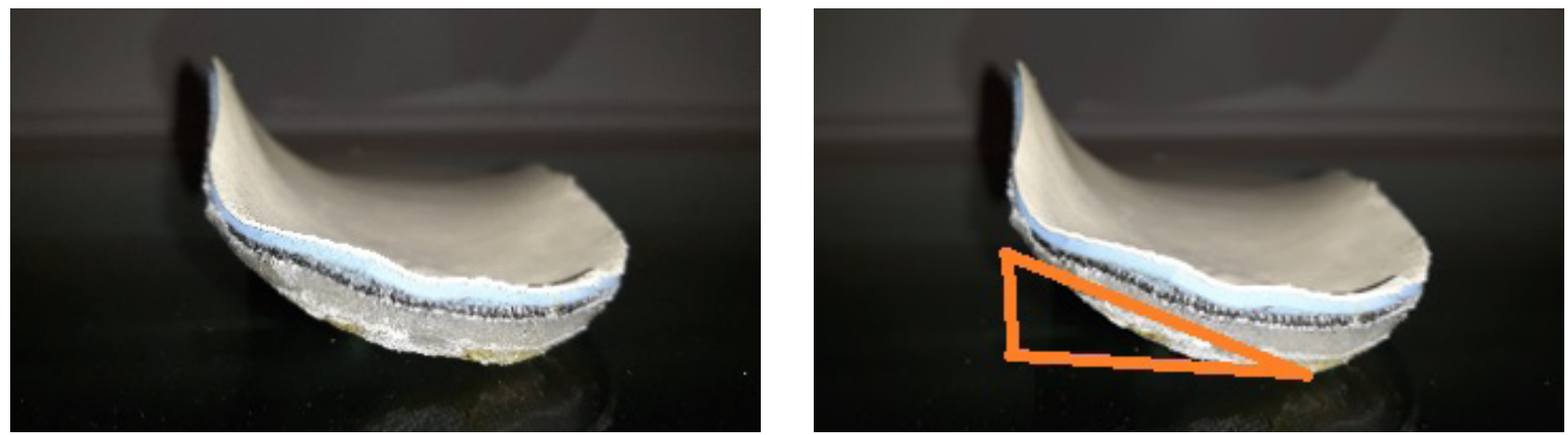

Figura 3. Imagen de la izquierda, ortesis estabilizada. A la derecha esquema del hueco a rellenar por material duro de alta densidad.

\section{Modificación técnica TAD. Medial y lateral TAD}

En este artículo se presentan dos modificaciones de la TAD. Una manual (Moisés Pardos, DP, comunicación personal, 6 de octubre de 2018) y otra a través de cuñas de alta densidad (Daniel García, DP). En el TAD tradicional, tras la exploración habitual y la obtención del patrón de la ortesis, se procede al recorte del material de la ortesis y a su calentamiento posterior previo a la adaptación en directo a través de la máquina de vacío, tal y como describieron Céspedes y Dorca'. Puede observarse un esquema de la técnica tradicional en la Figura 1.
Ambas modificaciones consisten en crear una cuña intrínseca en la ortesis, durante el enfriado de la resina, de tal manera que en el modo manual mediante una presión con la palma de la mano en la cara plantar e interna (medial TAD) o externa (lateral TAD), se crea un plano inclinado en la ortesis a nivel de talón, tal y como puede observarse en la Figura 2. Una vez enfriada la resina y estabilizada la plantilla, se procede al relleno y posteo con material duro de alta densidad del espacio en triángulo recto creado entre la plantilla y el suelo, ya sea medial o lateral, como se observa de forma esquemática en la Figura 3. 

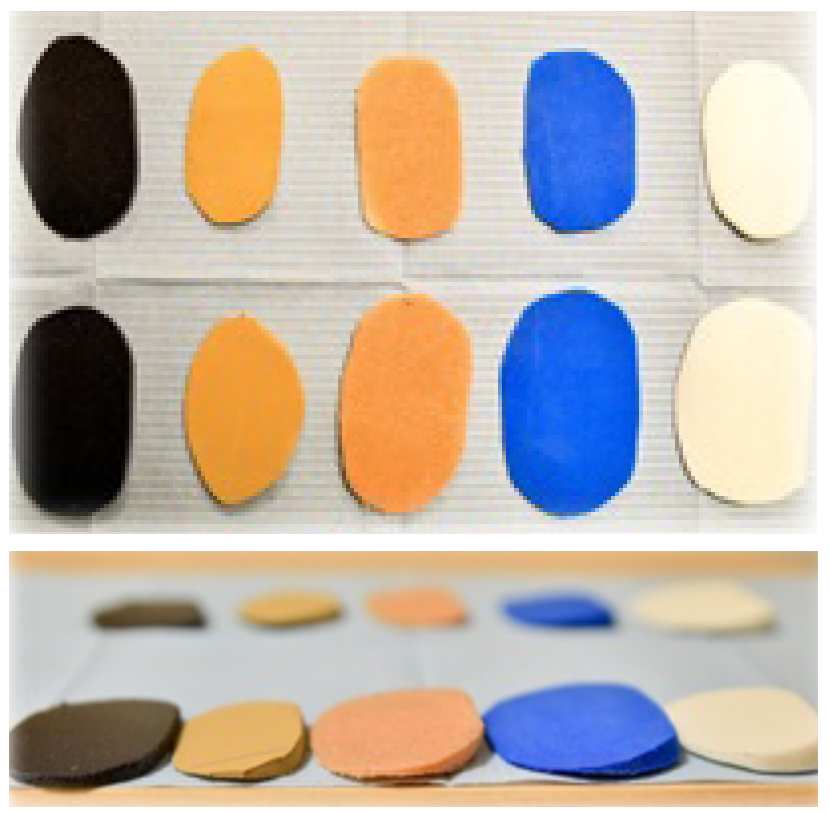

Figura 4. Visión cenital y perspetiva anteroposterior de las cuñas.

Daniel García, podólogo, propone una segunda modificación consistente en una manipulación de los soportes plantares mediante la aplicación de piezas de distintas medidas, en función del diagnóstico establecido y de la cantidad de corrección que se desea establecer. Se trata de cuñas biseladas, donde el mayor grosor de la pieza varía entre 1 centímetro y 0.5 para reducirse a cero en su parte opuesta, como puede observarse en la Figura 4. El tamaño puede variar en función de la medida del pie a tratar, y el material está considerado de alta densidad.

La aplicación de la cuña se realiza mediante pegado con tira adhesiva en la zona del talón donde se desee incidir, siendo aconsejable aplicarla al menos en los dos tercios mediales del retropie si se trata de un Medial, y en un tercio para un Lateral y pidiendo al paciente que se ponga de pie realizando así un molde en carga controlada, como puede observarse en la Figura 5.

Una vez realizada la carga sobre el soporte plantar durante aproximadamente 30 segundos, pueden utilizarse compresas de frío para terminar la adaptación que podría requerirse en función del diagnóstico. Para finalizar la ortesis ambas modificaciones, al igual que la técnica tradicional, requieren de maniobras de estabilización en antepié (Figura 5) y retropié con relleno y posteo medial o lateral de material duro de alta densidad.

En el caso clínico que se presenta se comparó el comportamiento cinemático de una ortesis TAD tradicional con una ortesis TAD modificada de forma manual. En la prueba se realizaron 4 capturas del movimiento para valorar los cambios cinemáticos en las situaciones de caminar descalzo
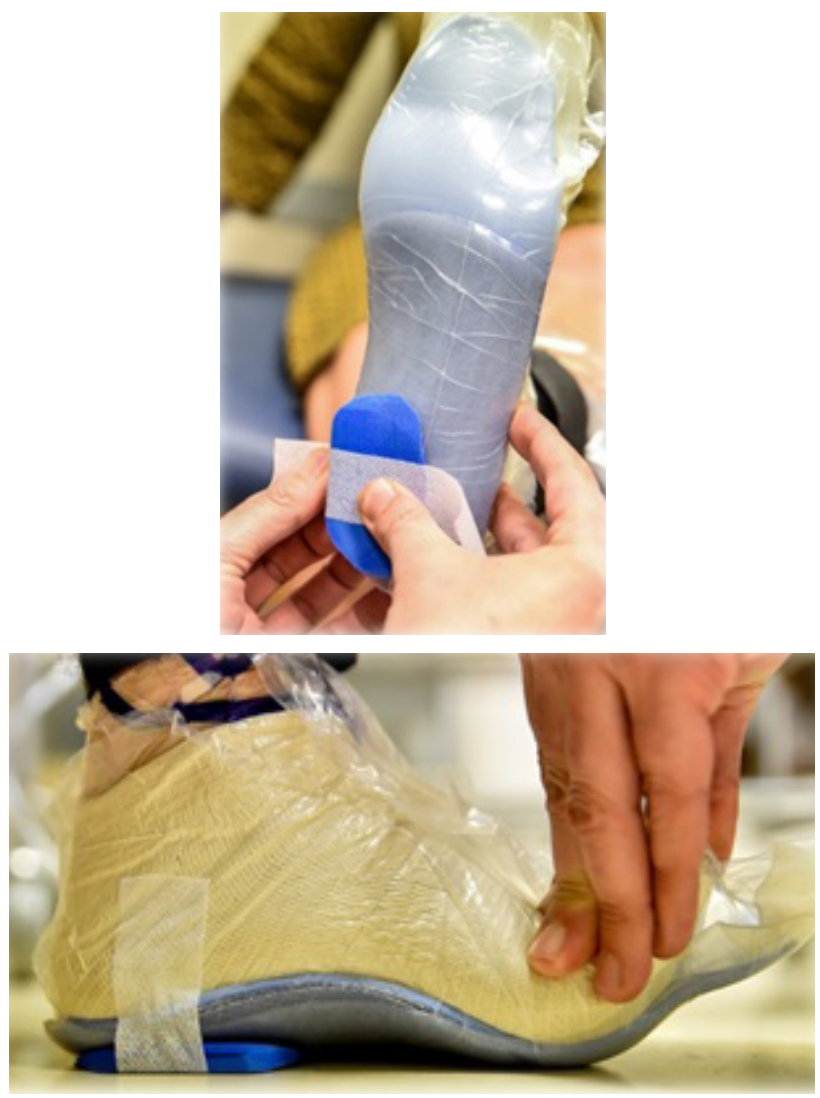

Figura 5. Visión plantar (izquierda) y medial (derecha) de la aplicación de las cuñas.

(DES), caminar calzado con deportiva (CS), caminar calzado con deportiva con ortesis Medial TAD (MEDIAL TAD) y caminar calzado con ortesis TAD tradicional (TAD). Se utilizó un sistema óptico de análisis formado por 9 cámaras infrarrojas Optitrack 9 y software Motive de ${ }^{\circledR}$ Natural Point Inc. ${ }^{2}$, que fijan su objetivo sobre marcadores reflectantes solidarios a segmentos óseos monitorizados. En cada una de las capturas se registran los valores de 10 pasos, traduciéndose gráficamente en un único registro representado por el valor medio, con el objetivo de eliminar la variabilidad individual, tal y como aconsejan algunos autores ${ }^{23-25}$. Los grados de rotación del eje Y están referenciados a la "posición de cero rotaciones (OR)" que vemos en la Figura 6.

Respecto a la AMT, en el plano frontal (Figura 7), el pie derecho descalzo (DES) inicia el apoyo en el suelo en una posición $0.5^{\circ}$ más supinada que la posición $\mathrm{OR}$, rotando en pronación $0.6^{\circ}$ hasta $x=12$, donde alcanza la posición máximamente pronada y se mantiene aproximadamente constante hasta el final de la fase de apoyo. El pie izquierdo descalzo inicia el choque de talón con el pie en una posición $5^{\circ}$ más supinada que la posición $\mathrm{OR}$, experimentando una pronación progresiva neta en fase de apoyo de aproximadamente $3.3^{\circ}$ hasta alcanzar la posición máximamente pronada en $x=50$, en una 


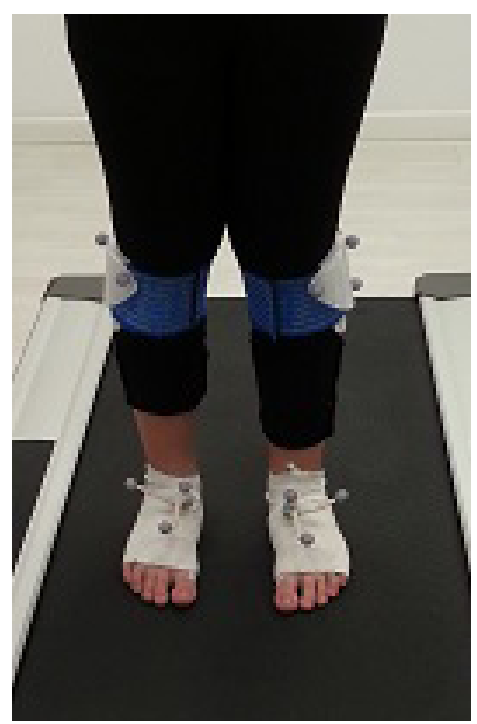

Figura 6. Posición de "cero rotaciones" (OR), a partir de la cual se referencian los valores cuantitativos de rangos articulares analizados y que coincide con el valor 0.0 del eje de abscisas de los gráficos.
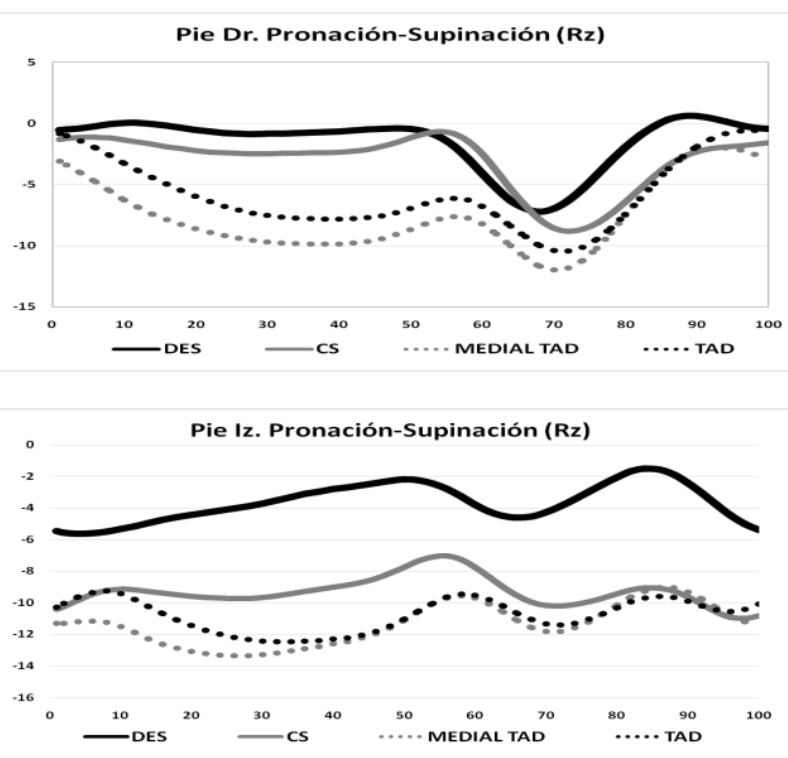

Figura 7. Rotación AMT en plano frontal (Rz). Eje horizontal, porcentaje del ciclo de la marcha. Eje vertical, grados relativos de rotación $\mathrm{Rz}$. Sentido ascendente de pronación y descendente de supinación. El valor 0 del eje vertical, corresponde con la posición OR de AMT, aproximadamente coincidente con la posición de la AMT al medir la PRCA (DC- $7^{\circ}$ VG, IZ-8 VG).

posición $2.2^{\circ}$ más supinada que la posición de $0 \mathrm{R}$. El rango pronador del pie derecho no superó los $0.6^{\circ}$, y en pie izquierdo alcanzó los $3.3^{\circ}$. Podría decirse que aunque el rango pronador del pie izquierdo es mayor que el derecho no alcanza
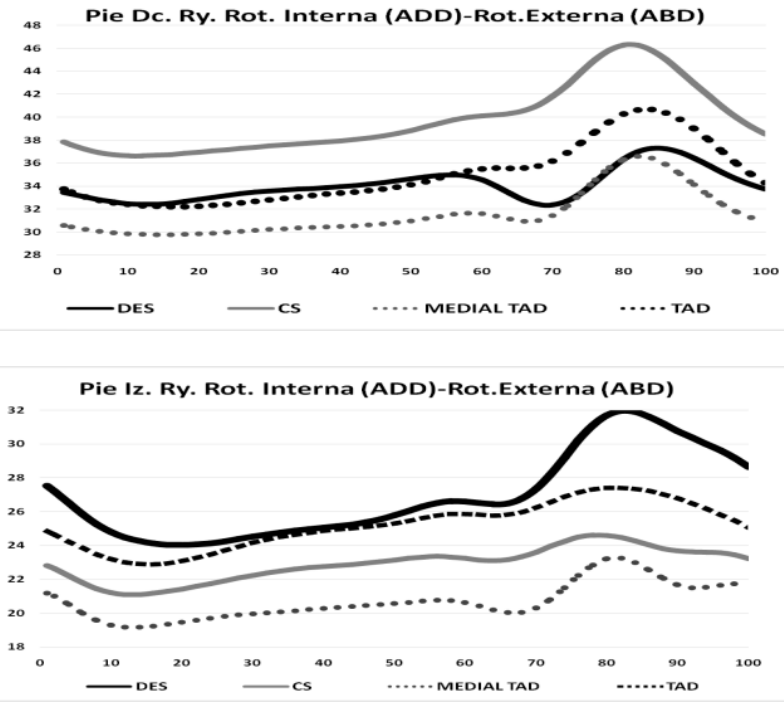

Figura 8. Rotación AMT en plano transverso (Ry) (Ángulo de Fick). Eje horizontal, porcentaje del ciclo de la marcha. Eje vertical, grados relativos de rotación Ry. Sentido ascendente de rotación externa (abducción) y descendente de rotación interna (aducción).

la posición máximamente pronada del pie derecho, aunque en estática el IPP del pie izquierdo indicaba una mayor pronación. La incorporación del cazado sin ortesis (CS) reduce entre $4^{\circ}(x=10)$ y $6^{\circ}(x=[20-50])$ la posición máximamente pronada del modo descalzo en pie izquierdo, pero solo $2^{\circ}$ en pie derecho entre $x=[15-45]$. El uso de ortesis supinadora mediante MEDIAL TAD eleva a $10-11^{\circ}$ la corrección en el pie izquierdo entre $x=[20-40]$ y entre 8 y $10^{\circ}$ en pie derecho entre $x=[15-50]$. La ortesis supinadora mediante TAD tradicional (TAD), corrige $2-3^{\circ}$ menos que la MEDIAL TAD en pie derecho durante toda la fase de apoyo y repite el registro de la plantilla MEDIAL TAD en la segunda mitad de la fase de apoyo en pie izquierdo, si bien tras el contacto de talón $(x=0-10)$, la ortesis $\mathrm{TAD}$ tradicional registra una rotación contradictoria en sentido pronador que podría interpretarse como un efecto inverso de la ortesis, situándose en una posición $2^{\circ}$ más pronada que con la ortesis MEDIAL TAD.

En plano transverso (Ry) de la AMT (Figura 8), tras el choque de talón se produce una rotación interna (aducción) del pie de aproximadamente $2-4^{\circ}$ en pie izquierdo y más suave de apenas $2^{\circ}$ en pie derecho hasta $x=15$. A partir de este momento ambos pies experimentan una rotación externa (abducción) progresiva de $2-3^{\circ}$, hasta el final de la fase de apoyo en $x=60$. El pie derecho descalzo (DES) refleja valores de abducción del pie $8^{\circ}$ superiores al izquierdo. El calzado (CS) rota externamente el pie derecho aproximadamente $4^{\circ}$ e internamente $3^{\circ}$ el izquierdo. La plantilla TAD tradicional (TAD) repite prácticamente el registro de pie descalzo en ambos pies. La plantilla MEDIAL TAD registra la posición de menor abducción. 


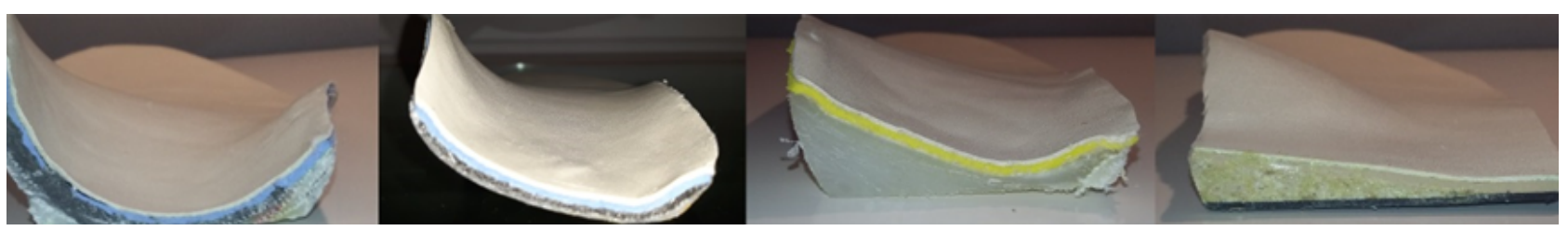

Figura 9. Corte transversal de tercio proximal del soporte plantar con corrección medial. De izquierda a derecha, ortesis con TAD tradicional, Ortesis con Medial TAD, Medial Heel Skive, cuña supinadora posterior de Lelièvre.

\section{DISCUSIÓN}

El marcador utilizado para la medición de los grados de rotación de la AMT tiene un diseño que envuelve el tubérculo del escafoides y permite su utilización en el interior del calzado sin crear interferencia ${ }^{2}$. Los resultados en cuanto a la reducción del valor de máxima pronación de AMT al incorporar el calzado $\left(4-6^{\circ}\right)$ son comparables a la investigación de $\operatorname{Pardos}^{2}\left(4.9^{\circ}\right)$ en pie izquierdo, pero no en pie derecho donde los valores de $2^{\circ}$ pondrían en evidencia la mayor afectación de la desviación en pie derecho, incluso harían replantear la obligación de comprobar el carácter rígido de la lesión. La reducción del valor de máxima pronación al usar las dos ortesis Medial TAD (hasta $11^{\circ}$ ) y TAD tradicional (hasta $8^{\circ}$ ) fue superior a la investigación de referencia ${ }^{2}\left(7.3^{\circ}\right)$. El control de pronación de la ortesis sí fue efectivo en ambos pies, no solo en el izquierdo (como sucedió con el calzado), pero sí más intenso en el izquierdo, lo cual reforzaría la idea de la mayor afectación del pie derecho. La diferencia con la investigación similar referida ${ }^{2}$ podría ser debida a que en aquella se trató de una muestra de 24 sujetos. Quizás el sujeto de este artículo representaría un caso extremo. Moraleda y Mubarak ${ }^{13}$ objetivaron un desplazamiento medial significativo del escafoides, Sinha y cols. ${ }^{14}$ y Books y cols. ${ }^{15}$ informaron de una mejora significativa en la angulación del astrágalo y escafoides, mediante mediciones radiológicas. Los rangos de pronación de la AMT fueron pequeños, $3.3^{\circ}$ (pie izquierdo) o casi nulos, $0.6^{\circ}$ (pie derecho), inferiores a los $7.27^{\circ}$ reportados por Pardos ${ }^{2}$ en una población de pies planos flexibles, y muy por debajo de $20.3^{\circ}$ reportados por Lundberg y cols. ${ }^{26}$, los $17.7^{\circ}$ de Ouzoniam y cols. ${ }^{27}$ en una investigación sobre 10 especímenes o los $13.5^{\circ}$ de Arndt y cols. con marcadores intracorticales ${ }^{28}$. Leardini y cols. ${ }^{29}$ y McWilliams y cols. ${ }^{30}$ encontraron rangos de rotación eversora de $2.8^{\circ}$ y $2.5^{\circ}$ respetivamente, pero referidos al segmento calcáneo, que a su vez rotó aproximadamente $10^{\circ}$, resultando finalmente un valor similar a los $13.5^{\circ} \mathrm{de}$ Arndt y cols. ${ }^{28}$. Los rangos de rotación reportados por Pardos ${ }^{2}$ utilizando un sistema de referencia global, el mismo utilizado en este caso clínico, resultarían claramente inferiores pero superiores al caso clínico que nos ocupa, lo cual apoyaría la idea de encontrarnos en un pie altamente pronado.

En la primera fase de contacto $(x=0-10)$, la ortesis TAD tradicional registró en pie izquierdo una rotación contradictoria en sentido pronador que podría interpretarse como un efecto inverso de la ortesis, similar al detectado en otras investiga- ciones $^{2}$ para el marcador de retropié. Este efecto quedaría anulado en el pie izquierdo al usar el MEDIAL TAD. Una posible explicación a estos hallazgos que podrían ser casuales y que merecerían un estudio clínico representativo sería el diseño de las ortesis. Al realizarse un corte transversal de las distintas ortesis (Figura 9) se observa la creación de un plano inclinado en la ortesis MEDIAL TAD de resina similar al TMHS o a la cuña supinadora posterior de Lelièvre, y ausente del perfil curvilíneo de la ortesis con el TAD tradicional.

De las observaciones cinemáticas del caso clínico expuesto, puede concluirse que el Medial TAD mejoró la adaptación del talón al suelo en el primer contacto del pie izquierdo y la posición de toda la fase de apoyo del pie derecho respecto de la ortesis confeccionada con TAD tradicional en plano frontal, y redujo el ángulo de Fick en ambos pies del sujeto estudiado. Este segundo hallazgo sería especialmente interesante en pie derecho con una mayor abducción del mismo, que podría explicar el distinto comportamiento de ambos pies en plano frontal con un pie derecho que "aterriza" en el suelo en una AMT casi máximamente pronada, para mantenerse en ella durante toda la fase de apoyo.

Una de las limitaciones del estudio es la monitorización del individuo durante la marcha sobre tapiz rodante, pero este elemento potencialmente distorsionador fue una constante en los tres modos de captura. No obstante, la reproducibilidad del sistema es considerada satisfactoria en comparación de otros sistemas ${ }^{31}$. Otra de las limitaciones es que los resultados no serían extrapolables y los hallazgos podrían ser casuales o quizás únicos lo que indicaría la necesidad de realización de un ensayo clínico representativo.

Finalmente, de las observaciones cinemáticas del caso clínico expuesto, puede concluirse que el MEDIAL TAD mejora la adaptación del talón al suelo en el primer contacto del pie izquierdo y la posición de toda la fase de apoyo del pie derecho respecto de la ortesis confeccionada con TAD tradicional en plano frontal y reduce el ángulo de Fick en ambos pies.

\section{CONFLICTO DE INTERESES}

Los autores no presentan conflictos de interés relevantes con el presente artículo

\section{FINANCIACIÓN}

No existen fuentes de financiación públicas o privadas en la realización del presente estudio. 


\section{BIBLIOGRAFÍA}

1. Céspedes T, Dorca A, Concustell J, Sacristán S, Céspedes M, Sánchez G. Técnica de Aplicación Directa (TAD) de ortesis sobre el pie: a propósito de varios casos clínicos. Rev Esp Podol. 1999;10(6):325-39.

2. Pardos M. Eficacia del tratamiento conservador ortésico en el pie plano flexible infantil. Rev Esp Pod. 2018;29(1):2-12. DOI: 10.20986/revesppod.2018.1507/2018

3. Thewlis D, Bishop C, Daniell N, Paul G. Next-generation low-cost motion capture systems can provide comparable spatial accuracy to high-end systems. J Appl Biomech. 2013;29(1):112-7. DOI: 10.1123/jab.29.1.112.

4. Betsch $M$, Wild $M$, Johnstone $B$, Jungbluth $P$, Hakimi $M$, KühImann $B$, et al. Evaluation of a novel spine and surface topography system for dynamic spinal curvature analysis during gait. PLoS One. 2013;8(7):e70581. DOI: 10.1371/journal.pone.0070581.

5. McGinley JL, Baker R, Wolfe R, Morris ME. The reliability of three-dimensional kinematic gait measurements: a systematic review. Gait Posture. 2009;29(3):360-9. DOI: 10.1016/j.gaitpost.2008.09.003.

6. Kobrick RL, Carr CE, Meyen F, Domingues AR, Newman DJ, Jacobs SE. Using Inertial Measurement Units for Measuring Spacesuit Mobility and Work Envelope Capability for Intravehicular and Extravehicular Activities. 63 ${ }^{\text {rd }}$ International Astronautical Congress, Space Life Sciences Symposium, Life Support and EVA Systems Session. Naples, Italy; 2012.

7. McCulloch MU, Brunt D, Vander Linden D. The effect of foot orthotics and gait velocity on lower limb kinematics and temporal events of stance. J Orthop Sports Phys Ther. 1993;17(1):2-10. DOI: 10.2519/ jospt.1993.17.1.2.

8. Mündermann A, Nigg BM, Humble RN, Stefanyshyn DJ. Foot orthotics affect lower extremity kinematics and kinetics during running. Clin Biomech (Bristol, Avon). 2003;18(3):254-62. DOI: 10.1016/s02680033(02)00186-9.

9. Genova JM, Gross MT. Effect of foot orthotics on calcaneal eversion during standing and treadmill walking for subjects with abnormal pronation. J Orthop Sports Phys Ther. 2000;30(11):664-75. DOI: 10.2519/ jospt.2000.30.11.664.

10. Eng JJ, Pierrynowski MR. The effect of soft foot orthotics on three-dimensional lower-limb kinematics during walking and running. Phys Ther. 1994;74(9):836-44. DOI: 10.1093/ptj/74.9.836.

11. Williams DS, Mcclay DI, Baitch SP. Effect of inverted orthoses on lower-extremity mechanics in runners. Med Sci Sports Exerc. 2003;35(12):2060-8. DOI: 10.1249/01.MSS.0000098988.17182.8A.

12. Nigg BM, Stergiou P, Cole G, Stefanyshyn D, Mündermann A, Humble N. Effect of Shoe Inserts on Kinematics, Center o Pressure, and Leg Joint Moments during URNG. Med Sci Sports Exerc. 2003;35(2):314-9.

13. Moraleda L, Mubarak SJ. Flexible flatfoot: differences in the relative alignment of each segment of the foot between symptomatic and asymptomatic patients J Pediatr Orthop. 2011;31(4):421-8. DOI: 10.1097/BPO.0b013e31821723ce.

14. Sinha S, Song HR, Kim HJ, Park MS, Yoon YC, Song SH. Medial arch orthosis for paediatric flatfoot. J Orthop Surg (Hong Kong). 2013;21(1):37-43. DOI: 10.1177/230949901302100111.
15. Bok SK, Kim BO, Lim JH, Ahn SY. Effects of custom-made rigid foot orthosis on pes planus in children over 6 years old. Ann Rehabil Med. 2014;38(3):369-75. DOI: 10.5535/arm.2014.38.3.369.

16. Blake RL. Inverted functional orthosis. J Am Podiatr Med Assoc. 1986;76(5):275-6. DOI: 10.7547/87507315-76-5-275.

17. Root ML, Orien WP, Weed JH, Hughes RJ. Biomechanical Examination of the Foot, Volume I. Los Angeles, CA: Clinical Biomechanics Corporation; 1971. p. 24.

18. Whitman R. Observations of forty-five cases of fiat-foot with particular reference to etiology and treatment. Boston Med Surg. 1888;118:598.

19. Shuster RO. A history of orthopedics in podiatry. J Am Podiatry Assoc. 1974;64(5):332-45. DOI: 10.7547/87507315-64-5-332.

20. Kirby KA. Foot and Lower Extremity Biomechanics IV: Newsletters, 2009-2013 Precision Intricast. Payson, Arizona: Precision Intricast, Inc; 2014. p .55-6.

21. Kirby KA, Green DR. Evaluation and Nonoperative Management of Pes Valgus. In: Steven DeValentine Dir. Foot and Ankle Disorders in Children. New York: Churchill Livingstone; 1992. p. 295-327.

22. Kirby KA. The medial heel skive technique. Improving pronation control in foot orthoses.J Am Podiatr Med Assoc. 1992;82(4):177-88 .DOI: 10.7547/87507315-82-4-177.

23. Kneip A, Gasser T. Statistical tools to analyze data representing a sample of curves. Annals of Statistics. 1992;20(3):1266-305. DOI: 10.1214/ aos/1176348769.

24. Sadeghi H, Mathieu P, Sadeghi S, Labelle H. Continuous curve registration as an intertrial gait variability reduction technique. IEEE Transactions on Neural Systems and Rehabilitation Engineering. 2003;11(1):24-30. DOI: 10.1109/TNSRE.2003.810428.

25. Sadeghi H, Allard P, Shafie K, Mathieu P, Sadeghi S, Prince F, et al. Reduction of gait variability using curve registration. Gait Posture. 2000;12(3):257-64. DOI: 10.1016/s0966-6362(00)00085-0.

26. Arndt A, Wolf P, Liu A, Nester C, Stacoff A, Jones R, et al. Intrinsic foot kinematics measured in vivo during the stance phase of slow running. J Biomech. 2007;40(12):2672-8. DOI: 10.1016/j.jbiomech.2006.12.009.

27. Lundberg A, Svensson OK, Bylund C, Goldie I, Selvik G. Kinematics of the ankle/foot complex--Part 2: Pronation and supination. Foot Ankle. 1989;9(5):248-53. DOI: 10.1177/107110078900900508.

28. Ouzounian TJ, Shereff MJ. In vitro determination of midfoot motion. Foot Ankle. 1989;10(3):140-6. DOI: 10.1177/107110078901000305.

29. Leardini MG, Benedetti F, Catani L, Simoncini S, Giannini A. Anatomically based protocol for the description of foot segment kinematics during gait. Clinical Biomechanics (Bristol, Avon). 1999;14(8):528-36. DOI: 10.1016/S0268-0033(99)00008-X.

30. McWilliams BA, Cowley M, Nicholson DE. Foot kinematics and kinetics during adolescent gait. Gait Posture. 2003;17(3):214-24. DOI: 10.1016/S0966-6362(02)00103-0.

31. Marín J, Marín JJ, Blanco T, de la Torre J. Gait Analysis in a Box: A System Based on Magnetometer-Free IMUs or Clusters of Optical Markers with Automatic Event Detection. Sensors 2020;20(12):3338. DOI: 10.3390/ s20123338. 